\title{
Controlled HHG with a Sub-Cycle mJ-Level Parametric Waveform Synthesizer
}

\author{
Roland E. Mainz, ${ }^{1,2, *}$ Yudong Yang, ${ }^{1,2}$ Giulio Maria Rossi, ${ }^{1,2}$ \\ Fabian Scheiba, ${ }^{1,2}$ Giovanni Cirmi, ${ }^{1,2}$ and Franz X. Kärtner ${ }^{1,2}$ \\ ${ }^{1}$ Center for Free-Electron Laser Science, Deutsches Elektronen-Synchrotron DESY, \\ Notkestraße 85, 22607 Hamburg, Germany \\ ${ }^{2}$ Physics Department and The Hamburg Centre for Ultrafast Imaging, University of Hamburg, \\ Luruper Chaussee 149, 22761 Hamburg, Germany \\ *roland.mainz@desy.de
}

\begin{abstract}
We present high-harmonic generation (HHG) driven with a sub-cycle mJ-level parametric waveform synthesizer. The HHG yield and spectral shape can be controlled by varying the carrier-envelope phase and the relative phase in the synthesizer channels.
\end{abstract}

OCIS codes: (320.0320) Ultrafast optics; (340.7480) X-rays, soft x-rays, extreme ultraviolet (EUV)

High harmonic generation (HHG) in gas medium [1] is the work horse for generating coherent extreme ultraviolet (XUV) radiation in laser laboratories and the only process currently demonstrated to generate attosecond pulses. In the past few decades, the ultrafast community has invested substantial efforts into HHG related researches, such as cutoff photon energy extension and conversion efficiency enhancement. The cut-off photon energy increases when longer wavelength driver pulses are used. However, the HHG conversion efficiency drops drastically due to the unfavorable wavelength scaling factor [2]. In order to further optimize the HHG process, non-sinusoidal customized waveforms have been proposed [3,4]. Those proposed waveforms allow in theory to extend the cut-off photon energy and/or efficiency. One possible approach to generate high-energy sub-cycle waveforms in the infrared region lies in optical parametric synthesis [5].

In our laboratory, we have recently built a parametric waveform synthesizer where two already almost single-cycle pulses in the infrared (IR) and near-infrared (NIR) spectral range are combined into a 0.5-cycle pulse with central wavelength of $1.8 \mu \mathrm{m}$ [6]. The setup allows controlling the waveform of the electric field transient. Here, we present our first results concerning the generation of quasi-continuum waveform-controlled high harmonics. This preliminary results suggest the possibility to control the total yield and the spectral shape of the HHG by adjusting both the relative phase (RP) and the carrier-envelope phase (CEP) of the two channels, thus shaping the synthesized waveform.
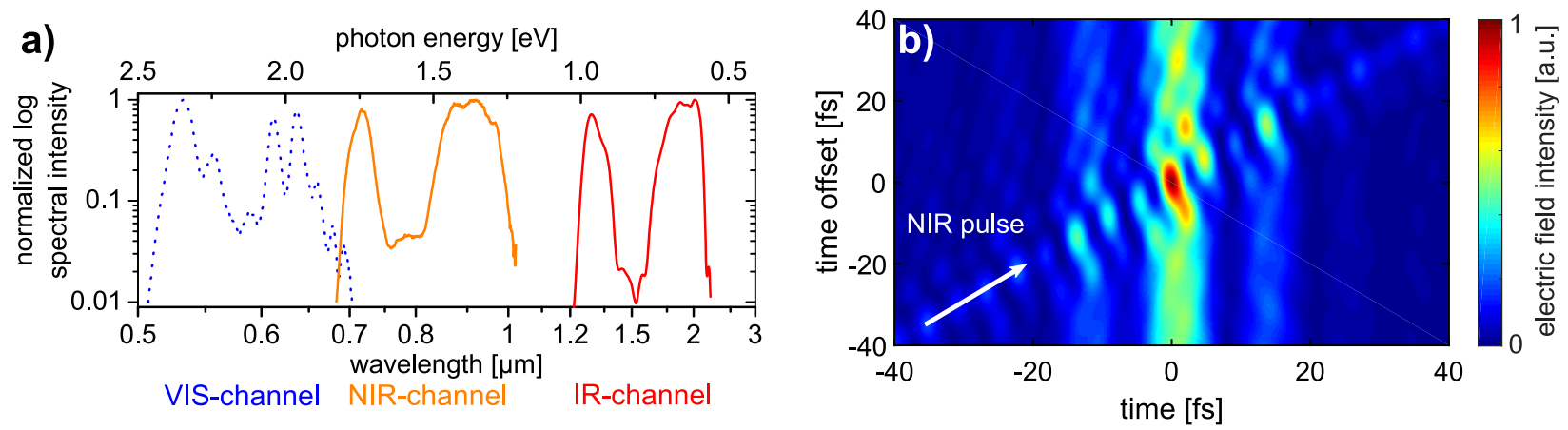

Fig. 1. (a) Output spectrum of the parametric waveform synthesizer, IR channel (red) and NIR channel (orange) are used for driving HHG, the visible channel (blue dashed) is currently under construction; (b) $I(t)$ profiles of the synthesized fields obtained by numerically applying a different $\mathrm{RP}$ (or time offset) to the two measured $I(t)$ of the individual synthesizer channels (each with CEP $=0^{\circ}$ ).

The parametric waveform synthesizer consists of two different three-stage optical parametric amplifiers (OPAs), which are pumped by a commercial Ti:Sapphire laser amplifier $(20 \mathrm{~mJ}, \sim 150 \mathrm{fs}, 800 \mathrm{~nm}, 1 \mathrm{kHz}$ ). The laser output is used to generate (via an OPA) CEP-stable and controlled white-light seed pulses and to pump the OPAs. Feedback loops are implemented to stabilize and control the CEP and the RP in the two OPA channels (both about $200 \mathrm{mrad} \mathrm{rms}$ ). The spectra of the two channels covers the 680-970 nm region (NIR) and the 1250-2200 nm region (IR) respectively (see 
Fig. 2). By finely adjusting the dispersion and the time delay between two channels, $\sim 3.4$ fs FWHM pulses can be obtained at the HHG source. A spherical mirror $(\mathrm{f}=500 \mathrm{~mm})$ focuses the synthesized pulses into a $4 \mathrm{~mm}$ long gas cell filled with argon (backing pressure $400 \mathrm{mbar}$ ). The focal points of the two channels are carefully adjusted to overlap with each other. At the interaction point the pulse energy is $75 \mu \mathrm{J}$ for the NIR and $450 \mu \mathrm{J}$ for the IR-channel. An XUV-grating spectrometer (McPherson, 251MX) with XUV CCD-camera (Andor, Newton) is used to measure the HHG spectrum. The fundamental frequency is filtered by a $200 \mathrm{~nm}$ thick aluminum filter. We observe that the HHG spectrum obtained with the NIR channel alone exhibits the typical discrete lines of odd harmonic orders. After adding a portion of the IR channel, the HHG spectrum changes significantly to a quasi-continuum.
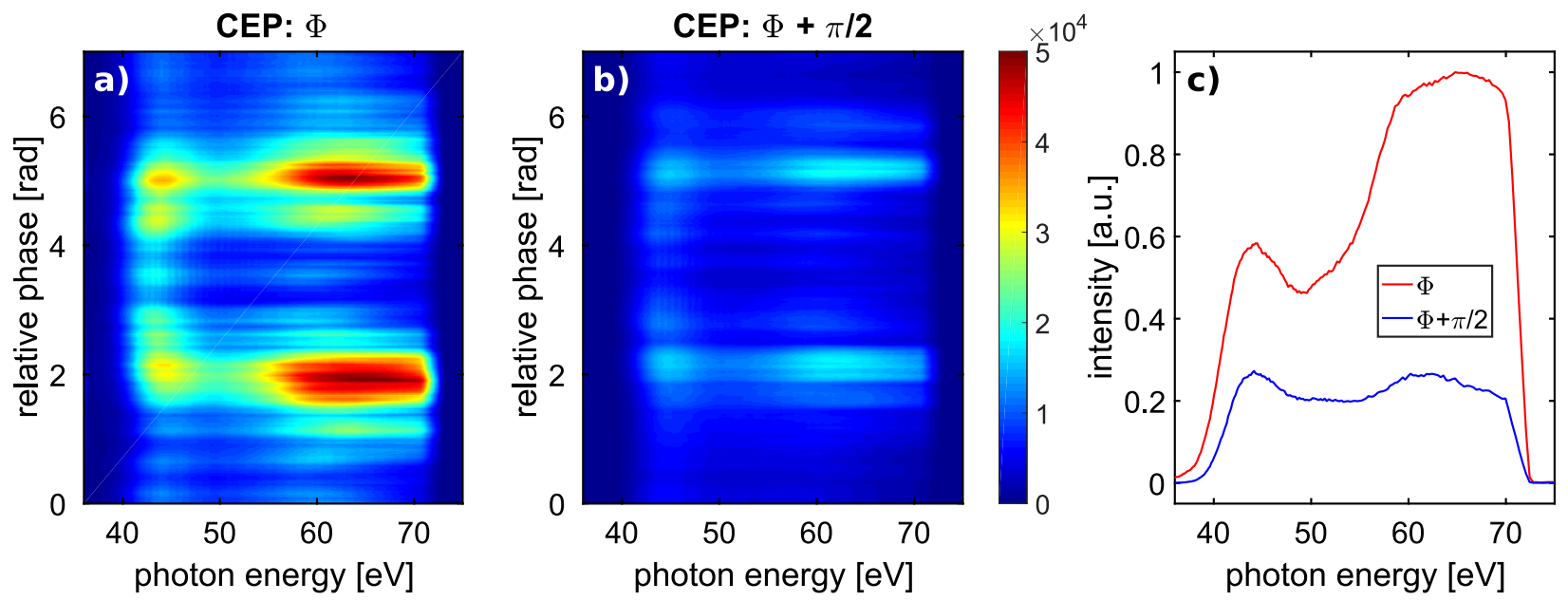

Fig. 2. (a,b) Dependence of the HHG spectrum upon changes of the RP between the two synthesizer channels for two different seed pulse CEP values. Each spectrum was acquired with an integration time of $10 \mathrm{~ms}$ and averaged over 50 samples. For a relative CEP $=\Phi(\mathbf{a})$ the overall HHG yield is higher and the relative intensity of the high photon energy part is increased with respect to CEP $=\Phi+\pi / 2$ (b) for any RP value. (c) Spectra corresponding to the RPs that maximize each HHG yield for both CEP values.

To showcase the possibility to control the HHG spectral shape by the driver waveform, we scan the CEP of the seed pulses as well as the RP for different CEP values. From Fig. 2 it is clear that both the seed CEP and the RP have strong effects on the HHG spectral shape. Different values of CEP and RP allow to vary the overall HHG yield by more than ten-fold. Moreover, different CEP values leads to different spectral shapes, once the RP is chosen to maximize the yield, as shown in Fig. 2(c). These preliminary results do not offer a straightforward interpretation and, due to the complicated electric field of the synthesized pulses, one should not expect simple $\pi$-periodicity in the evolution of the HHG spectrum once the CEP and the RP are changed. Moreover, our current RP piezo-actuator does not allow us to perform a complete RP-scan (cross-correlation). In the results we show here, it is clear that the seed CEP and the RP can be used as knobs to control the HHG spectrum, proving as well the stability of the system. The current effort is focused on the generation of HHG at higher photon energies via higher ionization-potential gasses as well as on the realization of an attosecond-streaking apparatus capable of characterizing both the synthesized field and the generated HHG pulses. After further optimization of the synthesis control system (increased RP scanning range, OPA pump timing lock, etc.) and implementation of the third visible channel, our parametric waveform synthesizer promises to generate nonsinusoidal waveforms optimized for efficient HHG cut-off generation and direct isolated attosecond pulses production.

\section{References}

1. A. McPherson et al., JOSA B 4, 959 (1987)

2. E. L. Falcão-Filho et al., Opt. Express 13, 11217-11229 (2009)

3. C. Jin et al., Nature Communications 5, 4003 (2014)

4. L. E. Chipperfield et al., Phys. Rev. Lett. 102, 063003 (2009)

5. O. D. Mücke et al., IEEE J. Sel. Top. Quantum Electron. 21, 8700712 (2015)

6. G. M. Rossi et al., submitted to CLEO PR 2018 